\title{
Der Konvent über die Zukunft der EU als verfassungsgebende Versammlung
}

Teija Tiilikainen

\section{Einleitung}

Angesichts der bevorstehenden großen Erweiterung der Europäischen Union (EU) kam es in ihren Mitgliedstaaten Anfang der 2000-er Jahre zu einer breiten Diskussion über die Zukunft der Union. Im Brennpunkt der Diskussion standen der Charakter der Union und ihres Vertragssystems, dessen verständlichere Gestaltung und Bürgernähe weithin angemahnt wurden.

Das endgültige Ausmaß des Änderungsbedarfs bestätigte der Europäische Rat im Dezember 2001 in Laeken mit dem Beschluss, einen breit angelegten Sonderkonvent einzuberufen, der die angestrebten umfassenden Änderungen der EU-Verträge ausarbeiten sollte. ${ }^{1}$ Mit den Änderungen sollte die EU demokratischer, offener und effizienter werden. Ein Hauptmittel zum Erreichen dieser Ziele war die Reform der EU-Verträge durch Verstärkung ihres Grundgesetzcharakters. Wegen dieses zentralen Auftrags und des großen Umfangs der vorgesehenen Vertragsänderungen bezeichnete man den Konvent, der im Frühjahr 2002 seine Tätigkeit aufgenommen hatte, als »Verfassungskonvent $«$.

Das Konventsmodell zur Erarbeitung der Vertragsänderungen heranzuziehen, hatte sowohl eher prinzipielle als auch rein praktische Gründe. So war eine Regierungskonferenz als Gremium zur Änderung der EU-Verträge wegen ihres undemokratischen und intransparenten Charakters schon länger kritisiert worden. Als Beispiel für eine offenere Vorbereitungsarbeit auf breiterer Basis diente das Modell des Konvents zur Erarbeitung der Charta der Grundrechte, in dem neben den Regierungen der Mitgliedstaaten auch deren Parlamente sowie die Europäische Kommission und das Europäische Parlament vertreten waren. Wie bei diesem ersten Konvent wollte man auch mit dem Verfassungskonvent eine ausschließlich von den Regierungen getragene Verhandlungskonstellation und somit auch die

1 Die Bezeichnung EU-Verträge verweist hier und im Weiteren auf die ursprünglichen EG-Gründungsverträge und deren später geänderte Fassungen. 
Veto-Rechte einzelner Mitgliedstaaten umgehen und so ein ehrgeizigeres Abschlussergebnis ermöglichen.

Wegen der Art und dem Umfang seiner Agenda richteten sich an den Verfassungskonvent noch weitergehende Erwartungen. Viele erblickten in ihm sogar einen ordentlichen verfassungsgebenden Konvent, vergleichbar etwa mit der Philadelphia Convention in den USA 1787, aus der die Verfassung der Vereinigten Staaten hervorging. Diesem Denkansatz zufolge galt die Auffassung, beim Verfassungskonvent liege die verfassungsgebende Gewalt der gesamten Union, deren Verfassungsform er bestimmen solle.

In diesem Kapitel wird der Verfassungskonvent der EU aus eben diesem Blickwinkel seiner verfassungsgebenden Rolle betrachtet. Dabei stellen sich folgende Fragen: Inwieweit traf die Gleichsetzung mit früheren verfassungsgebenden Versammlungen zu und inwieweit unterschied sich der Konvent, der im Jahre 2003 seinen Entwurf zum Vertrag über eine Verfassung für Europa (VVE) vorlegte, von anderen bekannten verfassungsgebenden Versammlungen? Mit dem im Jahre 2009 in Kraft getretenen Vertrag von Lissabon wurde das Konventsmodell zu einem ständigen Vorbereitungsgremium für alle die Fälle, in denen seine Heranziehung wegen der Tragweite der Änderungen an den EU-Verträgen geboten scheint. Im Lichte der Erfahrungen werden in diesem Kapitel auch die strukturellen und funktionellen Schwächen und die Verbesserungserfordernisse des Konventsmodells als eines künftig satzungsgemäß einzusetzenden Instruments behandelt. Ganz zu Anfang aber werden das demokratische Wesen verfassungsgebender Versammlungen und dessen Einbindung in die früheren Phasen der europäischen Integration betrachtet.

\section{Verfassungsgebender Konvent}

Die Umstände der Entstehung moderner Verfassungen variieren stark. Mit dem Durchbruch der demokratischen Regierungsform begann man, die Einführung von Grundgesetzen mit der Forderung nach »Volksherrschaft« zu verknüpfen. Die Demokratie kann als direktes oder repräsentatives System umgesetzt werden. ${ }^{2}$ Als allgemeine Praxis hat sich herausgebildet, zur Vorbereitungsarbeit eines neuen Grundgesetzes eine spezielle Versammlung bzw. einen Konvent einzuberufen, dessen Zusammensetzung oder

2 Siehe z.B. Markku Suksi, Making a Constitution: The Outline of an Argument (Åbo Academy University, 1995), 53-94. 
Wahlverfahren den besonderen Charakter seines Mandats widerspiegelt. Über die Einberufung des Konvents zur Erarbeitung der Verfassung der USA zum Beispiel wurde im 18. Jahrhundert eine Volksabstimmung abgehalten. Später konnte über die Einsetzung einer verfassungsgebenden Versammlung entweder durch Wahlen oder einen Parlamentsbeschluss entschieden werden, wobei allerdings ein vom Parlament selber erarbeitetes Grundgesetz noch vom Volk gutgeheißen werden musste.

In der Geschichte der Europäischen Union gab es bereits früher Versuche, die Integration durch Erlass einer Verfassung auf eine grundgesetzliche Basis zu stellen. Sofort nach der Entstehung der ursprünglichen Europäischen Gemeinschaft für Kohle und Stahl (EGKS) wurde ihr Ausbau durch Verteidigungskooperation und stärkere föderative Strukturen angestrebt. ${ }^{3}$ Zur Erreichung dieser Ziele bevollmächtigten die damals sechs Mitgliedstaaten der EG die Gemeinsame Versammlung der gerade erst gegründeten EGKS, als ad-hoc Sonderversammlung zu fungieren und ein Grundgesetz für die zu gründende Europäische Politische Gemeinschaft zu entwerfen. ${ }^{4}$ In ihrer als Mandat dienenden Luxemburger Erklärung beschlossen die Außenminister der Mitgliedstaaten 1952 die Zusammensetzung der Sonderversammlung und die Leitvorgaben in Übereinstimmung mit den Zielen des zuvor im gleichen Jahr geschlossenen Vertrages über die Europäische Verteidigungsgemeinschaft (EVG). Auf deren Grundlage sollte die zu entwerfende Verfassung die Basis für eine politische Gemeinschaft in Form eines Bundesstaates oder Staatenbundes schaffen, deren Exekutive sich auf ein direkt zu wählendes ZweikammerParlament stützte und auf dem Prinzip der Gewaltenteilung beruhte.

Diese erste verfassungsgebende Sonderversammlung der EG gründete ein Vorbereitungsgremium, das sich selbst »Verfassungsausschuss" nannte. Der auf einer Föderativordnung beruhende Entwurf zu einem Vertrag über die Satzung der Europäischen (Politischen) Gemeinschaft (EPG) ${ }^{5}$ wurde bereits wenige Monate später an die Regierungen der Mitgliedstaaten übergeben, jedoch trat er wegen der veränderten politischen Verhältnisse nie in Kraft. Der Entwurf des Vertrags über die Satzung war in seiner Rechts- und Bestätigungsform weiterhin ein Staatsvertrag, doch basierte

3 Rita Cardozo, »The Project for a Political Community«, in Roy Pryce (Hrsg.), The Dynamics of European Union (Routledge, 1989).

4 In der Luxemburger Erklärung (10. September 1952) mit dem Mandat ist die Rede sowohl von einer »Politischen Gemeinschaft« als auch einer »Politischen Autorität«.

5 Entwurf zu einem Vertrag über die Satzung der Europäischen (Politischen) Gemeinschaft (EPG), 1953. 
er weitgehend auf dem Prinzip der Supranationalität und hätte die Kompetenzen der damaligen Europäischen Gemeinschaft für Kohle und Stahl (EGKS) beträchtlich erweitert. Der Vertragsentwurf verwies auch zum ersten Mal auf die Europäische Gemeinschaft als einen Zusammenschluss der Völker und Staaten.

Einen zweiten Versuch zur Schaffung einer Verfassung für die Europäische Gemeinschaft gab es Mitte der 1980-er Jahre, als das zum ersten Mal in Direktwahl gewählte Europäische Parlament nach einigem Hin und Her einen Entwurf eines Vertrages zur Gründung der Europäischen Union annahm. ${ }^{6}$ Mit der Arbeit am eigentlichen Verfassungsentwurf betraute das Parlament seinen eigenen Ausschuss für institutionelle Fragen, der hierfür gegründet wurde und mit der Vorbereitungsarbeit nach Weisung der Plenarversammlung des Parlaments begann. Für den vom Parlament verabschiedeten Gründungsvertrag war zur Inkraftsetzung ein Verfahren vorgesehen, bei dem eine Ratifizierung durch die Mehrheit der Mitgliedstaaten ausreichen würde, sofern diese Länder zwei Drittel der Bevölkerungszahl der Gemeinschaft repräsentierten. Trotz aktiver Bemühungen des Europäischen Parlaments kam der Vertrag als solcher nicht zustande, obwohl er bei der Entwicklung der Zuständigkeiten der Union und institutioneller Fragen langfristig noch eine starke Rolle spielen sollte. Im Jahre 1985 starteten die Mitgliedstaaten eine Regierungskonferenz über eine Reform der EU-Verträge. Die Agenda der Regierungskonferenz enthielt zwar auch die Entwicklung einer politischen Union, doch blieb das Ambitionsniveau in der Sache noch recht niedrig, und erst bei der Erarbeitung des Vertrags von Maastricht einige Jahre später kamen die Auswirkungen des vom Parlament vorgelegten Vertragsentwurfs voll zum Tragen. Die mit dem Vertrag von Maastricht geschaffene Rechtsgrundlage der EU beruhte jedoch weiterhin auf Staatsverträgen zwischen den Mitgliedstaaten, so dass eine Konstitutionalisierung auch damals nicht eingeleitet wurde.

\section{Der Europäische Verfassungsprozess im neuen Jahrtausend}

Anfang der 2000-er Jahre teilte die Frage des politischen Charakters der EU deren Mitgliedstaaten mindestens genauso stark wie Mitte der 1950-er Jahre, als die Gründung einer politischen Gemeinschaft scheiterte. Mit einer Rede an der Humboldt-Universität hatte der deutsche Außenminis-

6 Europäisches Parlament, »Entwurf eines Vertrages zur Gründung der Europäischen Union«, (14. Februar 1984). 
ter Joschka Fischer die Diskussion über die Zukunft der Union eingeleitet; sie enthielt - für die damaligen Verhältnisse - ausgesprochen freimütige und vorurteilsfreie Ansätze zur endgültigen Ausrichtung und zu den politischen Strukturen der Union. ${ }^{7}$ Die Themen im öffentlichen Diskurs zur Zukunft der Union betrafen die Form ihres politischen und rechtlichen Systems. In den meisten Diskursbeiträgen wurde dessen klarere Gestaltung und Demokratisierung gefordert, damit die Systeme ihre Effizienz und Legitimität auch nach der bevorstehenden großen Erweiterung der Union und der sich damit fast verdoppelnden Mitgliederzahl wahren könnten. Ein zentrales Motiv für den Reformwillen war auch die Erkenntnis, dass eine Reform der Union im damaligen EU-Vertragssystem angesichts der wachsenden Mitgliederzahl immer schwieriger würde. Laut seinem Mandat sollte der vom Europäischen Rat in Laeken mit breiter Basis eingesetzte Konvent daher die Prinzipien des gesamten Systems der EU-Verträge auch aus dieser Sicht überprüfen.

Obwohl sich in der Einberufung des Konvents keine Hinweise auf einen verfassungssetzenden Auftrag finden, ergibt sich die ihm zugewiesene Rolle aus den Details. Der Konvent sollte als von den Mitgliedstaaten unabhängiges Gremium arbeiten und selbst über seine Agenda und Arbeitsweise entscheiden. Zur Leitung der Arbeit des Konvents wurde ein nicht an die Mitgliedstaaten angebundenes Präsidium gebildet. Die Tagungen des Konvents sollten offen sein und mit Anhörung der Zivilgesellschaft arbeiten, wofür ein spezielles Diskussionsforum eingerichtet wurde. Der Europäische Rat von Laeken hatte dem Konvent zahlreiche Punkte zur Demokratisierung und effizienteren Gestaltung der Tätigkeit der Union aufgezeigt, darunter auch die Frage, ob die Vereinfachung der EU-Verträge und deren Neuordnung seinerzeit zur Annahme einer Verfassung der Union führen könnten. ${ }^{8}$ Dass die Arbeit des Konvents zu einer Vorlage über eine EU-Verfassung führen könnte, hatte ja auch schon der zum Vorsitzenden des Konvents ernannte ehemalige Präsident von Frankreich Valéry Giscard d'Estaing in seiner Eröffnungsansprache des Konvents angedeutet. ${ }^{9}$

7 Joschka Fischer, »Vom Staatenverbund zur Föderation - Gedanken über die Finalität der europäischen Integration« (»Humboldt-Rede«, 12. Mai 2000).

8 Erklärung von Laeken zur Zukunft der Europäischen Union (14.-15. Dezember 2001).

9 »Eingedenk all dieser Gegebenheiten hat der Europäische Rat auf seiner Tagung in Laeken die Einberufung des Konvents zur Zukunft Europas beschlossen, dem Sie angehören, und diesem die Aufgabe übertragen, die Reform der Strukturen Europas vorzubereiten und - wenn wir uns dazu fähig erweisen - den Weg in Richtung 
Die Mehrheit der EU-Mitgliedstaaten war allerdings nicht bereit, auf den Konvent die vollständige verfassungsgebende Gewalt zu übertragen, was im vorbereitenden Charakter der Arbeit des Konvents zum Ausdruck kam. Dies erforderte auch Art. 48 des damaligen EU-Vertrages, gemäß dem die EU-Verträge nur mit einstimmigem Beschluss der Mitgliedstaaten geändert werden konnten. Der Konvent sollte das Ergebnis seiner Arbeit den Mitgliedstaaten vorlegen, die es behandeln und dazu Beschlüsse auf einer Regierungskonferenz gemäß den EU-Verträgen fassen würden. Der Konvent stand somit im Einklang mit der Rechtsordnung der Union und stellte also - anders als die beiden früheren verfassungsgebenden Versammlungen - keine Abweichung davon dar.

Im Rückblick scheint es aber doch offensichtlich, dass die dem Konvent gesetzten rechtlichen Schranken, speziell das Gebot des vorbereitenden Charakters seiner Arbeit, seine politische Stoßkraft in keiner Weise beeinträchtigten. Diese Stoßkraft ergab sich erstens aus seinem unabhängigen Status, seinen eigenen Führungsstrukturen und seinen ehrgeizigen Zielsetzungen. ${ }^{10}$ Gleich nach Arbeitsbeginn des Konvents wurde klar, dass sein hoch angesehenes Präsidium sich ein Scheitern seines Auftrags, dessen Ausführung die europäische Öffentlichkeit anderthalb Jahre lang aufmerksam verfolgte, nicht leisten konnte.

Zweitens arbeiteten im Konvent an den angestrebten umfangreichen Änderungen der Union Vertreter des Europäischen Parlaments, die schon seit Langem eine stärkere Rolle der Union, der nationalen Parlamente und der Kommission angemahnt hatten. Besonders bei der erstgenannten Gruppe war klar, dass man dem Konvent ehrgeizige Ziele steckte und dementsprechende Ergebnisse erwartete, deren gründliche Überprüfung samt Neubewertung durch eine Regierungskonferenz nicht in Frage käme.

Zusammen mit der politischen Akzeptanz für das Vorhaben in vielen Mitgliedstaaten erklären auch diese mit der Struktur und der politischen Dynamik des Konvents verbundenen Faktoren, warum er sich schon in seiner Anfangsphase die Aufgabe setzte, das Vertragssystem der Union

auf eine Verfassung für Europa einzuschlagen«, Valéry Giscard d’Estaing ».Eröffnungsrede des Vorsitzenden V. Giscard d'Estaing vor dem Konvent zur Zukunft Europas« (26. Februar 2002).

10 Zur Auffassung des Präsidiums des Konvents von dessen Status als verfassungsgebende Versammlung siehe Jo Shaw, "What's in a Convention? Process and Substance in the Process of European Constitution-Building «, in Jo Shaw et al. (Hrsg.), The Convention on the Future of Europe: Working towards an EU Constitution (The Federal Trust, 2003), 51. 
gründlich zu reformieren - hin zu einer Verfassung. ${ }^{11}$ Gleichzeitig ergab sich ein Konsens dazu, dass die Regierungskonferenz im Anschluss an den Konvent die Vorlage des Konvents nicht wesentlich abändern solle.

Diese Auffassung gründete auch in der technisch anspruchsvollen Natur des Arbeitsergebnisses des Konvents. Wenn die neuen EU-Verträge erst einmal in die neue konstitutionelle Basis eingepasst wären, wäre die Einführung größerer Änderungen nicht mehr möglich, ohne dass das gesamte Gebäude zusammenbräche.

\section{Die konstitutionelle Agenda des Konvents}

Nachfolgend betrachten wir, inwieweit der Konvent von seiner inhaltlichen Leistung her als verfassungsgebende Versammlung bzw. Konvent gelten kann. Klar ist, dass der Konvent von der Art und Tragweite der umgesetzten Agenda her konstitutionelle Gewalt ausübenden Versammlungen gleichzusetzen ist. Die Agenda des Konvents umfasste alle Grundsatzfragen zu Charakter und Tätigkeit der EU - vom Aufbau der Zuständigkeitsverteilung zwischen EU und Mitgliedstaaten über das System der EU-Organe bis hin zu den Rechtsinstrumenten und schließlich zur Art des Rechts- und Vertragssystems selber. Über die zentralen Themen wurde auf den Plenartagungen des Konvents offen diskutiert, darüber hinaus aber auch auf den formellen bis informellen Vorbereitungssitzungen, die von den Mitgliedern und Mitgliedergruppen des Konvents organisiert wurden. Die offiziellen Vorbereitungsgremien des Konvents waren neben dem Präsidium die Arbeitsgruppen zu den zentralen Einzelthemen, die ein Abschlussdokument erstellten, das die Standpunkte ihrer Mitglieder zusammenfasste. Sie sollten dazu beitragen, einen Konsens im Konvent insgesamt herbeizuführen.

Der Umfang des vom Konvent wahrgenommen Auftrags ist auch aus dem bereits Ende Oktober 2002 vom Präsidium vorgelegten Vorschlag ersichtlich, das gesamte vorhandene Vertragssystem mit einem VVE zu ersetzen. ${ }^{12}$ Aus dem Vertragsentwurf geht hervor, wie weitgehend die Einzelheiten zur Tätigkeit der Union bis hin zu ihrem Namen vom Kon-

11 Siehe Europäischer Konvent, "Zusammenfassung der Schlussfolgerungen« (Präsidiumssitzung, Brüssel, 5. September 2002) www.european-convention. europa.eu (abgerufen am 11. April 2018).

12 Vorentwurf des Verfassungsvertrags (CONV 369/02). 
vent beurteilt werden sollten. ${ }^{13}$ Nur eine umfassendere Neubewertung der Kompetenzen der Union war in das Mandat des Konvents nicht aufgenommen worden, wenn auch zu einzelnen Zuständigkeiten diskutiert und für einige von ihnen im Abschlussdokument des Konvents eine Ausweitung vorgeschlagen wurde. ${ }^{14}$

Der grundsätzlichste Auftrag des Konvents bzw. die Umänderung der EU-Verträge in eine regelrechte Verfassung nahm während der Vorbereitungsarbeit unterschiedliche Formen an. Eine verständlichere Fassung und Vereinfachung des Systems der EU-Verträge waren von Anfang an auf der Themenliste des Konvents, und deren Umgestaltung in ein Dokument mit Verfassungscharakter rief keine größeren Widersprüche hervor. Schon die Erklärung von Laeken enthielt den Ansatz, über eine Neuordnung der EU-Verträge nachzudenken. Auch wurde in der Erklärung erwogen, ob man die EU-Verträge und die Bestimmungen anderer Verträge voneinander trennen und unterschiedliche Änderungs- und Ratifikationsverfahren schaffen solle.

Die Frage nach dem Charakter der zu erarbeitenden EU-Verfassung ist im Rückblick interessant, denn als Einzelfrage bestimmt sie am stärksten das Wesen des Konvents als ordentliche verfassungsgebende Versammlung und konstitutioneller Hoheitsträger. In den vom Präsidium erarbeiteten Schriftstücken sowie im »Penelope«-Arbeitspapier der Kommission zu einem konstitutionellen EU-Vertrag kam gegen das Jahresende 2002 mit Nachdruck der Gedanke auf, nach dem Verfahren gemäß den EU-Verträgen einen Vertrag in Kraft zu setzen, mit dem eine nach Charakter und Änderungsverfahren von dieser Logik abweichende Verfassung geschaffen würde. ${ }^{15}$ Für diese neu zu schaffende Verfassung sollte ein Änderungsverfahren gelten, das eine bestimmte Mehrheit der Mitgliedstaaten erforderte und somit einen Verzicht auf das Einstimmigkeitserfordernis der Mitgliedstaaten gemäß Artikel 48 der damaligen Verträge bedeutete. Außerdem sollte diese Verfassung für einzelne Punkte kürzere Änderungsverfahren vorsehen, auf die die bei normalen Änderungen üblichen schwerfälligen einzelstaatlichen Zustimmungsverfahren keine Anwendung fänden. In die

13 Im Vorentwurf des Verfassungsvertrags (CONV 369/02) werden als Alternativen Europäische Gemeinschaft, Europäische Union, Vereinigte Staaten von Europa und Vereintes Europa vorgeschlagen.

14 Als Beispiele seien die Kompetenzausweitung der Union auf die Energiepolitik oder internationale Verträge oder für das gemeinsame Handeln bei Terroranschlägen oder Naturkatastrophen (sog. Solidaritätsklausel) genannt.

15 CONV 369/02 und Vereinfachung der EU-Verträge und Ausarbeitung eines neuen Grundlagenvertrages, Sekretariat (CONV 250/02). 
Abschlussvorlage des Konvents zu einem VVE gelangte das auf einer Mehrheit beruhende Änderungsverfahren jedoch nicht. Dagegen sah sie das Konvent-Verfahren als Verfahrensweise bei allen umfangreicheren Vertragsänderungen vor. Die Annahme der endgültigen Änderungen bliebe jedoch dann einer Regierungskonferenz der Mitgliedstaaten vorbehalten. Der Europäische Rat wird in eine Lage eingreifen, wenn ein Fünftel der Mitgliedstaaten die Änderungen im Laufe von zwei Jahren nicht ratifiziert. Damit sollte verhindert werden, dass Ratifizierungsprobleme in einer kleinen Minderheit der Mitgliedstaaten automatisch die Verwirklichung der Vertragsänderungen blockieren.

Darauf, dass die neue Verfassung rechtlich auf eine neue Grundlage gestellt werden sollte, verweisen auch viele andere Details der Vorbereitungsarbeit. Wegen des Charakters der neuen Verfassung und auch angesichts der Notwendigkeit, das vom Konvent zu überarbeitende Vertragssystem überschaubarer zu machen, erhielt von den vorgelegten vertragstechnischen Mustern die Alternative Vorrang, bei der ein neuer Vertrag alle früheren EU-Verträge ersetzen würde und letztere somit hinfällig würden. Für diese Alternative sprach auch, dass nur sie die Möglichkeit bot, für die Union das angestrebte klar verständliche konstitutionelle Normenwerk zu schaffen. Diese Alternative gelangte auch in den abschließenden Vorschlag des Konvents und in den eigentlichen Verfassungsvertrag - trotz der damit verbundenen großen Herausforderungen. Die größte davon war die Situation in den Mitgliedstaaten, die dem neuen Vertrag aus diesem oder jenem Grund nicht zugestimmt hätten. In der neuen Lage hätte es keinerlei Verhandlungsspielraum mit der Möglichkeit gegeben, auch nur einzelne Bestimmungen der alten Verträge als Auffangvorschriften zu nutzen, weil diese Verträge aufgehört hätten zu existieren. Die Mitgliedstaaten hätten vor der Wahl gestanden, dem neuen Vertrag zuzustimmen oder die Union zu verlassen.

Der neuartige Typ der neuen Verfassung wurde auch in den Anfangsdiskussionen des Konvents zu den Zuständigkeiten der Union und zur Art der Kompetenzabgrenzung ausgearbeitet. In dieser Debatte zur Art der neuen Verfassung und in den dazugehörigen Unterlagen obsiegte eine im Vergleich zu den vorgenannten Ansätzen - vorsichtigere Herangehensweise. Die Zuständigkeiten der Union sollten auch weiterhin als von den Mitgliedstaaten übertragene Zuständigkeiten gelten, was die Rechtsbeziehung der Mitgliedstaaten und der Union unverändert belassen würde. Mit diesem Ansatz entstand im Konvent der Konsens, eine Art Liste der Zuständigkeiten auf der Grundlage der drei Hauptzuständigkeitsbereiche der Union zu definieren. Nur eine sehr kleine Minderheit der Mitglieder des Konvents wäre bereit gewesen, umgekehrt zu verfahren und der Union 
in der neuen Verfassung die Hoheit für die Zuständigkeiten zuzuweisen und die Kompetenzen der Mitgliedstaaten aufzulisten.

Um das System der Zuständigkeiten flexibler zu gestalten, unterstrich die Mehrheit des Konvents jedoch die Bedeutung der in den Verträgen enthaltenen sog. Flexibilitätsklausel (zum Beispiel Art.352, Vertrag über die Arbeitsweise der Europäischen Union). Diesen Vertragsvorschriften zufolge kann unter bestimmten Umständen eine Kompetenz der Union geltend gemacht werden, wenn ihre Ziele oder Tätigkeit dies erfordern.

Unabhängig von der Art und dem Änderungsverfahren der zu erlassenden Verfassung herrschte im Konvent deutlich die Auffassung vor, dass die Schaffung der Verfassung selbst nach dem normalen, in den EU-Verträgen vorgesehenen Verfahren bzw. nach Artikel 48, Vertrag über die Europäische Union, mit Einstimmigkeit der Mitgliedstaaten und unter Einhaltung der nationalen Zustimmungsverfahren erfolgen sollte. Da der Prozess auf politische Schwierigkeiten stoßen könnte und die EU sich noch dazu inmitten eines Vertragszustimmungs- und -ratifizierungsverfahrens erweitern würde, wurden im Konvent eventuelle alternative Verfahrensweisen zur Inkraftsetzung des Verfassungsvertrages erwogen. Im Konvent gab es eine von vielen, u.a. vom Vorsitzenden Giscard d'Estaing, mitgetragene Meinung, dass es möglich sein müsste, den Vertragsentwurf des Konvents mit einer deutlichen Mehrheit der Mitgliedstaaten und Ratifizierungen in Kraft zu setzen. Eine Minderheit einiger weniger Mitgliedstaaten sollte nicht in der Lage sein, den Vertrag zu Fall zu bringen. ${ }^{16}$ In die Konventsvorlage zu einem VVE wurde dies jedoch nicht übernommen, vielmahr sollte der Vertrag ihr zufolge dem üblichen Einstimmigkeits- und nationalem Zustimmungsverfahren unterworfen werden.

\section{Aufbau und Arbeitsweisen des Konvents}

In ihrer personellen Zusammensetzung unterschieden sich die Versammlungen, denen im Integrationsprojekt eine Art verfassungsgebender Rolle zugewiesen worden war, beträchtlich voneinander. War die Zusammensetzung der zwei ersten Versammlungen (1953 und 1984) rein politisch und repräsentativ, so basierte die Struktur des Verfassungskonvents von 2002 auf einer Repräsentation der zentralen politischen Institutionen der EU. Die Teilnehmerstruktur dieses Konvents entsprach der des kurz zuvor be-

16 Bruno de Witte, »The Process of Ratification of the Constitutional Treaty and the Crisis Options« (2004) EUI Working Paper LAW 16/2004, 4. 
endeten Grundrechtekonvents, der die Charta der Grundrechte der EU erarbeitete. Die gleiche Teilnehmerstruktur gilt auch für Konvente, die in Zukunft zur Erarbeitung von Änderungen der EU-Verträge einberufen werden (Vertrag über die Europäische Union, Art. 48, Abs. 3).

In der Erklärung von Laeken war die Zusammensetzung des Verfassungskonvents wie folgt festgelegt worden: für jeden der damals 15 Mitgliedstaaten je 1 Vertreter der Staats- oder Regierungschefs und je 2 Vertreter des nationalen Parlaments. Hinzu kamen 16 Mitglieder des Europäischen Parlaments und zwei Kommissionsvertreter. Die damaligen Bewerberstaaten der Union waren genauso wie die Mitgliedstaaten vertreten, konnten allerdings ein unter den Mitgliedstaaten erreichtes Einverständnis nicht blockieren. Zum Konvent gehörten ferner 12 Beobachter vom Wirtschafts- und Sozialausschuss, vom Ausschuss der Regionen und der Europäische Bürgerbeauftragte. Jedes ordentliche Mitglied hatte auch einen persönlichen Stellvertreter, der das Teilnahmerecht für alle Sitzungen hatte.

Bei der Einsetzung des Konvents war das Zuordnungsverhältnis der mitwirkenden Gruppen der Union (Vertreter von Regierungen und Parlamenten, Europäischem Parlament und Kommission) zu ihren Entsendern nicht definiert worden. Dies blieb im Ermessen der jeweiligen Entsender, und in der Zuordnungsweise kam es im Laufe der Arbeiten des Konvents auch zu Veränderungen. Am Anfang des Konvents ernannten viele Regierungschefs Personen außerhalb der Regierung oder Beamtenschaft zu ihren Vertretern, um so die Unabhängigkeit der Arbeit des Konvents zu betonen. ${ }^{17}$ Einige Mitgliedstaaten wie zum Beispiel das Vereinigte Königreich (Peter Hain) oder Belgien (Louis Michel) ernannten amtierende Minister zu ihren Vertretern. Als sich dann das Arbeitsergebnis des Konvents als offensichtlich sehr ambitiös erwies, ersetzten u.a. sowohl Deutschland (Joschka Fischer) als auch Frankreich (Dominique de Villepin) ihre früheren unabhängigen Vertreter durch ihre Außenminister. Die neuartige Sichtweise auf die Arbeit des Konvents schlug sich auch in der Praxis nieder, denn waren Vorlagen zunächst im Namen der Konventsmitglieder eingebracht worden, so geschah dies später auch im Namen von Mitgliedstaaten oder Gruppen von Mitgliedstaaten.

17 Vertreter der deutschen Regierung war Professor Peter Glotz, der französischen der ehemalige Europaminister Pierre Moscovici und der dänischen der Berater Henning Christophersen, der früher Minister in einer Regierung seines Landes und auch Kommissionsmitglied gewesen war. Die Verfasserin dieses Artikels war Vertreterin der finnischen Regierung ausschließlich aufgrund ihres wissenschaftlichen Hintergrundes. 
Auch bei den Vertretern der nationalen Parlamente gab es in ihrem Verhältnis zu ihren verbundenen Gruppen große Unterschiede, denn unter ihnen waren sowohl Persönlichkeiten in politisch oder institutionell herausgehobenen Positionen als auch sog. Hinterbänkler. Bei den Mitgliedern der nationalen Parlamente konnte das Repräsentativitätserfordernis auch problematisch werden, da die Mitarbeit im Konvent sehr detaillierte Sichtweisen zur Entwicklung des EU-Systems erforderte. In einigen EULändern mit einem parlamentarischen System hat das nationale Parlament außerhalb des parlamentarischen Dialogs keine selbständige Rolle. Eine Konstellation, in der Vertreter sowohl des Parlaments als auch der auf dessen Vertrauen angewiesenen Regierung als voneinander unabhängige Instanzen im internationalen Kontext agieren, ist in vielen Verfassungen nicht vorgesehen. ${ }^{18}$

Grundsätzlich galt in der Arbeit des Konvents die Gleichstellung seiner ordentlichen Mitglieder unabhängig von ihrer Hintergrundinstitution. Alle im Konvent vertretenen Gruppierungen hatten einen Vertreter im Präsidium, und alle Konventsmitglieder konnten gleichberechtigt Vorlagen einbringen und bei Bedarf abstimmen. Die Arbeitsgruppen, die als offizielles Vorbereitungsforum des Konvents dienten, standen allen Konventsmitgliedern offen.

Neben der offiziellen Funktionsstruktur des Konvents entstand eine starke informelle Parallelstruktur, in deren Dynamik sich die wichtigsten politischen Trennlinien widerspiegelten. Zu einer Koordination und Herausbildung gemeinsamer Standpunkte unter den Vertretern der Regierungen oder der nationalen Parlamente kam es nur sehr wenig, und im politischen Gefüge des Konvents erlangten die Fraktionen des Europäischen Parlaments oder verschiedene Gruppierungen der Mitgliedstaaten je nach Sachfrage eine bedeutendere Rolle. Da die Konventsmitglieder größtenteils aus der Parteipolitik stammten, boten die auf EU-Ebene etablierten Parteigruppierungen eine ideologische wie administrative Plattform zur Erarbeitung eines gemeinsamen Standpunkts. Obwohl die Vorbereitungsarbeit in deren Rahmen nicht die gesamte umfangreiche Agenda oder alle Mitglieder des Konvents erfasste, kann diese parteipolitische Dimension dennoch als ein bedeutender Teil der Tätigkeit des Konvents gelten. ${ }^{19}$

18 Zur Beteiligung der nationalen Parlamente siehe Katrin Auel und Thomas Christiansen, "After Lisbon: National Parliaments in the European Union«, (2015) 38 West-European Politics 261.

19 Siehe auch Ben Crum, »Politics and Power in the European Convention«, (2004) 24 Politics 1, 4. 
Ein weiteres gewichtiges politisches Instrument in der Arbeit des Konvents waren die Interessengruppen "gleichgesinnter" Mitgliedstaaten, darunter als eine der am festesten etablierten die deutsch-französische Achse. ${ }^{20}$ Gemeinsame Initiativen mehrerer Länder zu zentralen Agendafragen halfen, einen breiteren Konsens herbeizuführen. ${ }^{21}$ Im Konvent entstand auch eine überaus eng geflochtene Koalition kleiner und mittelgroßer Mitgliedstaaten, deren gemeinsame Agenda den Schwerpunkt auf Fragen des institutionellen Systems und des politischen Machtgefüges der Union setzte. Was diese Koalition besonders nahe zusammenrücken ließ, war die gemeinsame Besorgnis wegen der Art und Weise, in der das Präsidium des Konvents die Behandlung der institutionellen bzw. einer der umstrittensten Fragen lenkte. Im Unterschied zur sonstigen Agenda des Konvents mit ihrem offenen Verhandlungsmodus sowohl in den Plenarsitzungen als auch in den als Entwurfsinstrumenten dienenden Arbeitsgruppen fand die Debatte zum politischen Machtgefüge in der Union weniger offen und ganz ohne Vorbereitung durch Arbeitsgruppen statt. Dieses Manko der Vorarbeiten bestärkte die Vertreter der kleinen Mitgliedstaaten in ihrem Misstrauen zu dieser Frage und in ihrer Kritik an den Vorlagen, die das Präsidium des Konvents zum politischen Machtgefüge der Union machte.

Wie bei früheren Änderungen der EU-Verträge waren die Fragen zum institutionellen System und zur politischen Machtausübung in der Union einer der umstrittensten Sachkomplexe des Konvents. Zwischen den großen und kleinen Mitgliedstaaten kam es zu beträchtlichen Meinungsunterschieden, besonders was die Umwandlung des Europäischen Rates in ein offizielles Organ der Union und den Übergang zu einem System mit einem ständigen Präsidenten des Europäischen Rates betraf. Weitere Streitfragen waren die angestrebte Verkleinerung der Zusammensetzung der Kommission und die Reform des Rotationsystems im Ratsvorsitz. Man war der Auffassung, dass diese Änderungen sowohl den Intergouvernementalismus der Entscheidungsfindung der Union verstärken als auch zugunsten der großen Mitgliedstaaten wirken würden. Als weniger umstritten erwies sich dagegen die Macht des Europäischen Parlaments, die der Konvent in seinem Vertragsentwurf sowohl hinsichtlich der Gesetzgebungs- und Haushaltsbefugnisse als auch der Zustimmung zu den Außenverträgen der Union zu erweitern vorschlug.

$20 \mathrm{Zu}$ den deutsch-französischen Beziehungen vgl. den Beitrag von Olaf Leiße in diesem Band.

21 Ein Beispiel für gemeinsame Initiativen mehrerer Länder ist ein Vorschlag zur gemeinsamen Europäischen Sicherheits- und Verteidigungspolitik (CONV 422/02). 
Das hybridartige politische Wesen der EU als Zusammenschluss von Mitgliedstaaten und Völkern zeigte sich in der Politik des Konvents. Die Mitgliederstruktur des Konvents gründete auf der Gleichstellung der Mitgliedstaaten, die sich in der gleichen Vertreterzahl pro Mitgliedstaat konkretisierte. Insofern entsprach der Konvent dem System der EU-Verträge und ihrem Geiste, der die Einstimmigkeit der Mitgliedstaaten bei Vertragsänderungen betont.

Die Unabhängigkeit des Konvents von den Mitgliedstaaten, seine Arbeitsweisen und Offenheit wiederum boten der europäischen Zivilgesellschaft mitsamt ihren politisch-ideologischen Trennlinien einen Freiraum und glichen somit die Größenunterschiede der Mitgliedstaaten aus. Die Politik des Konvents bildete eine vielförmige Gesamtheit, in der die Interessen der Mitgliedstaaten präsent waren, ohne jedoch eine dominante Rolle zu spielen. Die in die inoffizielle Vorbereitung des Konvents eng einbezogenen politischen Gruppierungen der EU-Ebene verliehen den europäischen Gesichtspunkten der Vorbereitung, u.a. auch der Stellung der Organe der EU, mehr Gewicht. Die Offenheit des Konvents ermöglichte auch eine rege Teilnahme von Akteuren der Zivilgesellschaft, die ihre Interessen über die formellen und informellen Einwirkungskanäle des Konvents einbrachten.

\section{Die Rolle des Konvents bei den Änderungen der EU-Verträge}

Letztlich konnte der Verfassungskonvent nicht in dem Ausmaß auf verfassungsgebende Befugnisse der EU zurückgreifen, als dass er in der Lage gewesen wäre, im normalen Vertragsänderungsverfahren eine grundsätzlichere Änderung der Rechtsgrundlage der Union herbeizuführen. Die abschließende Konventsvorlage zu einem VVE bedeutete eine inhaltlich breit angelegte, rechtlich jedoch eher moderate Änderung der damaligen Vertragsgrundlagen der Union. Der Konvent erreichte seine Ziele, das System der EU-Verträge zu vereinfachen und dessen Teile jeweils unterschiedlichen Abänderungsverfahren zuzuordnen. Voraussetzung für diese Vereinfachung war die Schaffung einer einheitlichen Rechtspersönlichkeit für die Union, die dem Vorschlag zugrunde lag. Der Vertragsentwurf des Konvents enthielt einen speziellen konstitutionellen Teil, der inhaltlich und der Form nach eher einer Verfassung als einem Staatsvertrag glich. Die Charta der Grundrechte mit der Bestätigung der bürgerlichen Grundrechte bildete im Aufbau des Entwurfs dessen zweiten gleichrangigen Teil. Der dritte Teil zu den Politikbereichen der Union war insgesamt am stärksten technisch ausgerichtet und für seine Abänderung war - ebenso 
wie bei einzelnen Abschnitten anderer Vertragsteile - ein einfacheres Verfahren vorgesehen.

Auch wurden die Ziele der Konventsmehrheit insofern erreicht, als bei der Behandlung des Konventsentwurfs auf der Regierungskonferenz 2003 bis 2004 daran nur marginale inhaltliche Veränderungen vorgenommen wurden, die Hauptlinien jedoch unangetastet blieben. Der von den EUMitgliedstaaten im Oktober 2004 unterzeichnete VVE war ein deutlicher Schritt hin zu einem Grundgesetz der Union - ohne Unterbrechung des rechtlichen Kontinuums der Union. Der gebilligte Vertrag sah - auch mit seinen zentralen inhaltlichen Wesensmerkmalen - wie ein Grundgesetz aus, war jedoch seiner Form nach ein Staatsvertrag zwischen den Mitgliedstaaten. Die Festschreibung seines Grundgesetzcharakters wäre in Zukunft auf dem Wege einer Reform seiner Änderungsverfahren oder einer vielleicht etwas schwierigeren Neuabgrenzung der Zuständigkeiten von Union und Mitgliedstaaten möglich gewesen, bei der die Geltung der Unionskompetenzen als von den Mitgliedstaaten abgetretene Zuständigkeiten abgeschwächt worden wäre.

Nachdem der VVE in den Referenden erst von Frankreich und dann der Niederlande 2005 jedoch abgelehnt worden war, wurden an ihm größere strukturelle Änderungen vorgenommen. Auf der Regierungskonferenz zur Ausarbeitung des Vertrags von Lissabon wurde dessen Grundgesetzcharakter in seiner alten Form wiederhergestellt und der Hauptteil des Vertragsinhaltes am Ende wie früher üblich über Änderungen am alten Vertragsbestand der Union verwirklicht. Die neue, besser überschaubare Struktur wurde also nur teilweise Wirklichkeit, doch der Hauptteil des Sachinhalts des Verfassungsvertrages kam ins Dasein, wenn auch nur in einer viel komplizierteren Form.

Auch das Konventsmodell selbst blieb im Vertrag von Lissabon als Vorbereitungsforum künftiger umfangreicher Änderungen an den EU-Verträgen erhalten (Vertrag über die Europäische Union, Artikel 48). Als nächstes folgt in diesem Kapitel eine Einschätzung der funktionellen Eignung des Konvents und seiner Zusammensetzung für diese künftige Aufgabe auch im Hinblick darauf, dass es bei künftigen Konventen nicht um die Ausübung konstitutioneller Befugnisse, sondern die herkömmlichere Vorbereitung von Staatsvertragsänderungen geht. Falls der Europäische Rat mit einfacher Mehrheit beschließt, ein Änderungsverfahren der EU-Verträge einzuleiten, so beruft er gemäß dem Vertrag von Lissabon einen Konvent mit dem Auftrag ein, die vorliegenden Änderungsvorschläge zu prüfen und Empfehlungen für eine spätere Regierungskonferenz zu geben. Der Konvent bedarf keiner Einberufung, wenn der Umfang der 
vorgeschlagenen Änderungen dies nicht erfordert. In diesem Fall muss das Europäische Parlament seine Zustimmung geben.

Mit seiner breiten Basis und Offenheit bietet das Vorbereitungsverfahren mit einem Konvent deutliche Vorteile. Die EU-Verträge sind inhaltlich von herkömmlichen Staatsverträgen weit entfernt, weshalb es sich anbietet, zur Vorbereitung von Änderungen und zu den Erwägungen im Vorfeld ein breiteres Forum als das rein zwischenstaatliche Verfahren zu nutzen. Für die Rolle der Organe der EU bei Änderungen der EUVerträge wurde ja gerade mit der Wichtigkeit des europäischen Blickwinkels und der Notwendigkeit, die relativ eingeschränkte zwischenstaatliche Wettbewerbskonstellation zu umgehen, argumentiert. ${ }^{22}$ Die Offenheit der Arbeitsweisen des Konvents ermöglichte die Beteiligung der europäischen Zivilgesellschaft und baute so Druck auf, an die Verhandlungsgegenstände auch unter dem Aspekt eines breiteren politischen Konsens heranzugehen. In den Lichtkegel des Konsensaspekts gerieten zum Beispiel die zahlreichen Versuche der Regierung des Vereinigten Königreichs zu verhindern, dass die Charta der Grundrechte der Union als gleichrangiger Bestandteil in die abgeänderten EU-Verträge aufgenommen wird.

Das Konvent-Verfahren als Bestandteil der Vorbereitung von Änderungen der EU-Verträge macht es erforderlich, die Mitgliederzusammensetzung und die Arbeitsverfahren des Konvents neu zu überdenken. Die in den EU-Verträgen verankerte Zusammensetzung gründet auf der institutionellen Repräsentation, deren Probleme letztlich auf die Legitimität des Konvents zurückwirken. Im Erfolgsfall könnte eine wirklich breite Repräsentationsbasis des Konvents bedeuten, dass die zentralen politischen Kräfte des Integrationsprozesses auch bei der Abfassung oder Änderung des rechtlichen Kerns der Union als Akteure legitimiert sind. Die Regierungen der Mitgliedstaaten gehören ex officio zu den zentralen Akteuren, weshalb für ihre Repräsentation im Konvent gemeinsame Spielregeln zu schaffen sind. Mit der Vertretung der Mitgliedstaaten kommt im Konvent das Prinzip ihrer Gleichstellung zum Tragen, weshalb es nicht sinnvoll sein kann, in den Mitgliedstaaten jeweils unterschiedlich auszulegen, wie niedrig- oder hochoffiziell diese Vertretung sei.

Die nationalen Parlamente sind im Laufe des letzten Jahrzehnts in vielerlei Zusammenhängen zu Akteuren auf EU-Ebene geworden. Haupt-

22 Shaw, "What's in a Convention?« (Fn. 10), 43-44. Zu EU-Vertragsreform und Rechenschaftspflicht siehe Christer Karlsson, »EU Treaty Reform and Accountability", in Sverker Gustafsson et al. (Hrsg.), The Illusion of Accountability in the European Union (Routledge, 2009). 
zweck dieser Entwicklung war es, das Demokratiedefizit der Union wettzumachen und die demokratische Kontrolle der Regierungen in EU-Angelegenheiten zu verstärken. Betrachtet man die Rolle der nationalen Parlamente jedoch vom Standpunkt der nationalen Verfassungen ebenso wie der Übersichtlichkeit des politischen Systems der EU und der politischen Zuständigkeitsverhältnisse, so ist diese Rolle nicht ganz unproblematisch. ${ }^{23}$ Erstens sehen die Verfassungsnormen der meisten politischen Systeme nur sehr wenig Raum für eine direkte internationale Rolle und Einflussnahme der Parlamente vor. In parlamentarischen Systemen können die Parlamente keine von der Regierung losgelösten Standpunkte zu EU-Fragen haben, und die nationalen Systeme kennen auch kein Auftreten des Parlaments als kollektiver Akteur. Im Hinblick auf eine direkte Mitsprache der nationalen Parlamente im EU-Kontext wurde auch angemerkt, dass sie die Macht- und Zuständigkeitsverhältnisse der beiden Ebenen des demokratischen Systems der Union unüberschaubar machen könnte.

Wird über eine Mitsprache der nationalen Parlamente eine Vertretung des nationalen politischen Spektrums angestrebt, die umfassender ist als nur über die Regierung, so müsste dies im EU-Kontext in anderer Weise als durch eine institutionalisierte Vertretung des nationalen Parlaments geschehen. Die Sache ist wichtig für das Funktionieren des politischen Systems der EU und sollte daher unmittelbar auf Unionsebene zur Erwägung gestellt werden. Ihre Bedeutung ist somit weittragender und wirkt sich nicht nur auf die Zusammensetzung des Konvents aus.

Die Vertretung des Europäischen Parlaments bedeutet im politischen Kontext der Union in der Regel eine Vertretung seiner Fraktionen entsprechend den politischen Kräfteverhältnissen. In der Praxis repräsentieren die Vertreter des Europäischen Parlaments also die Standpunkte der Parlamentsfraktionen, wie es ja auch im verfassungsgebenden Konvent der Fall war. Besonders die größten Fraktionen des Europäischen Parlaments fungierten als inoffizielle Vorbereitungsgremien des Konvents; es sollte daher erwogen werden, diese parteipolitische Dimension in künftigen Konventen so auszubauen, dass das System allen Fraktionen des Europäischen Parlaments die gleichen Einwirkungsmöglichkeiten gewährt. Die Zunahme der Befugnisse des Europäischen Parlaments wird also seine Position in künftigen Konventen verstärken, weshalb es begründet wäre, diese Einwirkungsrolle auch formalpolitisch zweckmäßig auszugestalten.

23 Auel und Christiansen, »After Lisbon« (Fn. 18). 
Ausschließlich aus der Sicht der Repräsentativität betrachtet, hängt die Rolle der Europäischen Kommission im Konvent davon ab, in welche Richtung sich ihr Verhältnis zum Europäischen Parlament entwickelt. Je unabhängiger die Rolle der Kommission aufgefasst wird, desto begründeter erscheint ihre Beteiligung an der Vorbereitung von Verträgen der Union. Die die Gewaltenteilung betonende Tendenz der letzten Zeit bietet somit eine stärkere Begründung für eine selbständige Rolle der Kommission auch bei der Arbeit an den EU-Verträgen. ${ }^{24}$

Der Verfassungskonvent entschied eigens über seine eigenen Arbeitsweisen, und angesichts seines Auftrags dürfte dieser Ansatz auch in $\mathrm{Zu}$ kunft gelten. Die hierzu am meisten debattierten Fragen waren der Begriff des Konsens und die Konsensbildung als Beschlussfassungsregel. In der Praxis funktionierte die Konsensbildung bei den meisten Sachkomplexen jedenfalls sehr gut, denn das Sekretariat erstellte Verhandlungsprotokolle mit einer Wiedergabe der vom Konsens abweichenden Standpunkte von Konventsmitgliedern oder Mitgliedergruppen. Der abschließende Konventsvorschlag zu einem neuen Vertrag enthielt dennoch einzelne Sachpunkte, die keinen breiteren Konsens widerspiegelten oder im Konvent noch nicht einmal Gegenstand einer gründlichen Debatte oder Wertung gewesen waren.

Ein ausreichendes Augenmerk muss bei künftigen Konventen deren Arbeitsweisen gelten, damit die allgemeinen politischen Zielvorgaben dieses breitgefächerten Vorbereitungsgremiums erreicht werden. Die Beibehaltung obskurer oder unklarer Verfahrensweisen beeinträchtigt das Ziel des Konvents, die demokratische Legitimität der Union zu fördern, und trägt somit auch nicht dazu bei, die angestrebte Wechselwirkung mit der europäischen Zivilgesellschaft zu intensivieren.

Wenn der Konvent für eine Ausweitung der Verfassungsprozesse der Union über den rein zwischenstaatlichen Ansatz hinaus sorgen soll, müssen seine Beteiligtenstruktur, Repräsentativität und politischen Verfahrensweisen auf dieses Ziel abgestimmt werden. Will man letztlich an einer Entscheidungsfindung aufgrund des Konsens unter allen Konventsmitgliedern festhalten, so müssten das Arbeitssystem bzw. die Arbeitsgruppen oder Fachausschüsse wie in einer echten parlamentarischen Struktur alle

24 Siehe Teija Tiilikainen, »Concepts of Parliamentarism in the EU's Political System«, FIIA Working Paper 108/2019; The Finnish Institute of International Affairs. Der Artikel kommt zur Schlussfolgerung, dass das politische System der EU in seiner jetzigen Form sich schon eher am für die USA typischen Modell der Gewaltenteilung orientiert als am ursprünglich angestrebten Modell des europäischen Parlamentarismus. 
Verhandlungsthemen erfassen. Außerdem müsste es klare Regeln dazu geben, nach was für einem Modus über die in diesen Vorbereitungsgremien erarbeiteten Vorlagen im Plenum des Konvents entschieden wird. Wenn andererseits die Rolle der Parteien auf EU-Ebene in die politische Vorbereitungsarbeit und Meinungsbildung des Konvents mit hineinkommt, sollte erwogen werden, wie die Rolle und Beteiligung der Regierungsvertreter im Verhältnis zur parteipolitischen Komponente abgesichert werden.

Als letztes bleibt die Frage zu überdenken, wie sich der Konvent als Vorbereitungsgremium zur Billigung der Vertragsänderungen selbst verhält, denn gemäß dem Vertrag von Lissabon gilt weiterhin das Gebot der Einstimmigkeit der Mitgliedstaaten und der Einhaltung der verfassungsmäßigen nationalen Zustimmungsverfahren. Eine Konstellation, in der eine knappe Mehrheit selbst des kleinsten Mitgliedstaates auf einem breiten europäischen Konsens beruhende Vertragsänderungen zu Fall bringen kann, ist kontraproduktiv, doch diesen Sachverhalt zu ändern erwies sich als besonders schwierig. Im verfassungsgebenden Konvent stand ein Referendum auf Unionsebene als eventuelles Verfahren zur Billigung von Änderungen der EU-Verfassung zur Diskussion. Von dieser Möglichkeit ist man jedoch noch weit entfernt; ein Referendum auf Unionsebene könnte bei umfassenden und grundsätzlichen Vertragsänderungen gleichwohl als Indikator für die unionsweite Akzeptanz einer Konventsvorlage dienen, bevor letztere in die Abschlussverhandlungen der Mitgliedstaaten und die nationalen Zustimmungsverfahren gelangt.

\section{Zusammenfassung}

Die bisherigen Versuche der EU, ihre Verträge von der Staatsvertragsgrundlage auf ein verfassungsartiges Regelwerk umzustellen, blieben unverwirklicht. Auf Grund seines überstaatlichen Charakters und seiner Unabhängigkeit gilt das Rechtssystem der Union schon seit langem als eine de facto verfassungsmäßige Rechtsordnung. Den Verfassungskonvent von Anfang unseres Jahrtausends kann man in vielerlei Hinsicht als eine ordentliche verfassungsgebende Versammlung ansehen, die auch ihre Möglichkeiten erwog, von der auf Grundlagenverträgen basierenden Unionsrechtsordnung abzuweichen und eine Verfassung einzuführen, die das Kontinuum dieser Rechtsordnung unterbrechen würde.

Dieser Versuch unterblieb jedoch, und der vorgelegte VVE, der eine gemäßigte Änderung des Grundcharakters des Vertragssystems bedeutet hätte, erlitt dasselbe Schicksal wie seine Vorgänger: Er scheiterte am Widerstand ausgesprochen knapper Mehrheiten in den Mitgliedstaaten. 
Das Konventsmodell als Vorbereitungsmechanismus für Änderungen der EU-Verträge blieb jedoch am Leben; sein Mehrwert als Verstärker der demokratischen Legitimität der Union sollte in Zukunft voll ausgeschöpft werden. Deshalb sollte noch vor der Einsetzung eines nächsten Konvents über dessen Zusammensetzung, die damit verbundenen Fragen der Repräsentativität und seine Arbeitsverfahren nachgedacht werden. Das Beispiel des verfassungsgebenden Konvents zeigt, dass die politische Basis des Konvents, seine Unabhängigkeit von Regierungen und die Beteiligung neuer Gruppen von Akteuren eine nur schwer zu bändigende politische Dynamik schaffen. Dabei ist darauf zu achten, dass diese Dynamik fest in demokratischen Verfahrensweisen verankert bleibt. 